\title{
BENEFÍCIOS DA FISIOTERAPIA AQUÁTICA NO PACIENTE COM ARTRITE REUMATÓIDE: REVISÃO DE LITERATURA
}

\author{
Viviane Santos Santana Fisioterapeuta graduada pela \\ Universidade Católica do Salvador.
Carlos Jose Vidal Euzébio Mestrado em Ciência da Família pela Universidade Católica do Salvador. Professor titular da Universidade Católica do Salvador. \\ Verena Loureiro Galvão
Mestrado em Medicina e Saúde Humana pela Fundação Bahiana para o Desenvolvimento das Ciências. Professor assistente da Universidade Católica do Salvador.

\begin{abstract}
Resumo
Introdução:A artrite reumatóide (AR) é uma doença inflamatória crônica que se caracteriza por poliartrite periférica que pode levar a deformidades, destruição articular e incapacidade funcional. A Fisioterapia Aquática diminui os efeitos da gravidade que resulta numa menor compressão nas articulações. Este trabalho tem como objetivo sistematizar o conhecimento a respeito da Fisioterapia Aquática no tratamento do paciente portador da AR. Metodologia: Trata-se de uma revisão de literatura realizada com acesso à base de dados Medline, biblioteca virtual Pubmed e consultas manuais nas referências dos artigos publicados entre 2000 a 2007. Foram incluídos estudos que abordassem a Fisioterapia Aquática no tratamento destes indivíduos, nos idiomas inglês e português. Foram excluídos artigos que relatassem a Fisioterapia Aquática no tratamento de portadores de AR submetidos a intervenção cirúrgica, ou que abordassem AR associada a outras doenças do colágeno, aqueles essencialmente farmacológicos e artigos referentes à balneoterapia. Oito artigos julgados relevantes localizados antes do período definido como critério de inclusão, também foram selecionados. Resultados: Após a pesquisa, foram encontrados 67 artigos. Destes, 14 foram incluídos no estudo. Predominantemente a metodologia foi composta de estudos intervencionais e de caso-controle. Conclusão: A Fisioterapia Aquática pode beneficiar os pacientes portadores de AR com intuito de aumentar a ADM, força muscular, capacidade cardiovascular, funcionalidade, equilíbrio postural, reduzir a dor e assim proporcionar uma melhora na qualidade de vida dos indivíduos com AR. Espera-se estimular a realização de novas pesquisas enfocando o tratamento aquático na AR.
\end{abstract}

Palavras-chave: Hidroterapia; Artrite reumatoide;Exercício aeróbico.

\section{BENEFITS OF THE AQUATIC PHYSICAL THERAPY IN PATIENT WITH RHEUMATOID ARTHITIS: LITERATURE REVIEW}

\begin{abstract}
Introduction: The rheumatoid arthritis (RA) is a chronic inflammatory disease that is characterized for peripheral polyarthritis that can carry the deformities, destruction articulate and functional inability. The aquatic physical therapy reducesthe effects of gravity that results in a lowercompressionjoints.Methods: This work has as goal systematize the knowledge concerning about of the in the patient's RA treatment bearer. It is a literature revision accomplished with access to the database Medline, virtual library Pubmed and manual consultations in the article references published between 2000 to 2007. They were included studies that boarded the aquatic physical therapy in the treatment of these individuals, in the english and portuguese languages. It were excluded articles that related the aquatic physical therapy in the bearers' treatment of submitted RA the surgical intervention, or that boarded RA associate to others collagen diseases, those essentially pharmacological and referring goods to balneotherapy. Eight important articles located tried before the defined period as inclusion criterion, also were selected. Results: After the research, they were found 67 articles. From this, 14 were included in the study. Predominantly the methodology was composed of interventional studies and of case-control. Conclusion: The aquatic physical therapy can benefit the patient with RA with aim of increasing ROM, muscle strength, cardiovascular capacity, functionality, balance postural, reduce the pain and thus provide one improves in the individuals life quality with RA. It waits to stimulate the new researches accomplishment focusing the aquatic treatment in the RA.
\end{abstract}

Keywords: Hydrotherapy;Rheumatoid arthritis; Aerobic exercise. 


\section{INTRODUÇÃO}

A artrite reumatóide (AR) é uma doença inflamatória crônica, sistêmica, auto-imune que se caracteriza por poliartrite periférica de forma simétrica, erosiva que pode levar a deformidades, destruição articular e incapacidade funcional. ${ }^{(2,13,20)}$ Apresenta manifestações articulares precedidas por fadiga, mialgia, febre, emagrecimento e manifestações extraarticulares. ${ }^{(12)}$ Em 1987, o Colégio Americano de Reumatologia estabeleceu sete critérios de classificação para o seu diagnóstico, sendo que quatro devem estar presentes por um período mínimo de seis semanas, indicando o paciente portador da $\mathrm{AR}^{(4,12,13,15,20)}$

Acomete cerca de $1 \%$ da população adulta, atingindo predominantemente mulheres, numa proporção de três mulheres para cada homem..$^{(4,5,13,20)}$ Pode ocorrer em qualquer faixa etária, freqüente entre os 30 a 50 anos, ${ }^{(5)}$ aumentando a prevalência com a idade. ${ }^{(20)}$ Há uma estimativa na qual anualmente 0,1 a0,2 a cada 1000 homens são atingidos e de 0,2 a0,4 a cada 1000 mulheres portadoras da AR. ${ }^{(5)}$

A AR não apresenta etiologia definida e pode estar relacionada à interação multifatorial envolvendo vírus, bactérias, fatores comportamentais, genéticos, distúrbios neuroendócrinos ou imunológicos. ${ }^{(2,5)}$ Alguns autores ${ }^{(5,20)}$ relatam a possibilidade de uma relação entre o vírus Epstein Barr e a $\mathrm{AR}$ em que 80\% dos pacientes com AR possuem anticorpos contra este vírus que é também ativador de células do tipo B levando a uma produção excessiva de imunoglobulinas até mesmo de fator reumatóide.

É de fundamental importância a prática de exercícios físicos para evolução dos pacientes com AR. Essas atividades devem ser realizadas com adequação e supervisão, no intuito de promover ao paciente uma melhoria na sua flexibilidade, na sua força, na função cardiovascular e na sua resistência, e, sobretudo, uma melhoria no desenvolvimento das suas habilidades diárias. ${ }^{(9)}$ Salienta-se que o meio aquático tem uma ótima indicaçãono tratamento desses pacientes, pois há uma diminuição dos efeitos da gravidade que resulta numa menor compressão sobre as articulações. Dessa forma, para evolução do quadro opta-se por realizar exercícios em piscina terapêutica ${ }^{(16)}$ que podem contribuir na correção de perda ou limitação das articulações, manter o trofismo muscular, favorecer o alinhamento e estabilidade articular. ${ }^{(2,5)}$ A Fisioterapia Aquática utiliza a piscina terapêutica numa temperatura entre $33^{\circ} \mathrm{e}$ $36^{\circ}$ que proporciona um meio ideal para a realização dos exercícios visando aliviar a dor e espasmos musculares, assim como melhorar o suprimento sanguíneo, equilíbrio, coordenação e postura. ${ }^{(2,16)}$ Vale ressaltar, entretanto, que ainda são poucos os estudos seqüenciados e 
randomizados em relação ao tratamento fisioterapêutico e, principalmente, os associados à piscina terapêutica e portadores de AR. Assim, o objetivo deste artigo foi sistematizar o conhecimento a respeito da Fisioterapia Aquática no tratamento do paciente portador da referida patologia.

\section{MATERIAL E MÉTODOS}

Trata-se de uma revisão de literatura realizada com acesso à base de dados Medline e à biblioteca virtual Pubmed. Além disso, consultas manuais nas referências dos artigos foram efetivadas. A busca foi limitada a estudos publicados no período compreendido entre 2000 a outubro de 2007, embora, oito artigos tenham sido de período anterior do referido por sua relevância. As palavras-chave utilizadas foram hidroterapia, artrite reumatóide, exercícios aquáticos, exercício aeróbico e seus correlatos em inglês e espanhol.

Os critérios de inclusão definidos para a seleção do presente trabalho foram artigos que abordassem a Fisioterapia Aquática no tratamento do paciente portador de AR. Os estudos que relatassem a Fisioterapia Aquática em pacientes com AR submetidos a intervenção cirúrgica, ou que abordassem AR associada a outras doenças do colágeno, aqueles essencialmente farmacológicos e artigos referentes à balneoterapia foram considerados como inadequados para a realização deste trabalho.

\section{RESULTADOS}

Somando-se todas as bases de dados foram encontrados 10 artigos na base de dados Medline, 61 na biblioteca virtual Pubmed e seis estudos em consultas manuais nas referências dos artigos. Após a leitura dos títulos, notou-se que alguns deles se repetiram e outros não preenchiam os critérios deste estudo. Foram selecionados 22 artigos para a leitura do resumo e excluídos os que não diziam respeito ao propósito do trabalho, sendo a maior quantidade de exclusões referentes à balneoterapia no tratamento da AR. Após a leitura dos resumos, foram selecionados 14 artigos, sendo 13 na língua inglesa e um em língua portuguesa que preencheram os critérios inicialmente propostos e foram lidos na íntegra. Predominantemente a metodologia foi composta de estudos intervencionais e de caso-controle.

As Tabelas 1, 2 e 3 sintetizam os resultados encontrados nos artigos selecionados para este estudo de revisão. 


\section{DISCUSSÃO}

A Fisioterapia Aquática aplicada ao tratamento do paciente portador da AR tem sido enfatizada na literatura. Observou-se que as evidências apontam para uma superioridade do tratamento aquático quando comparado com aquele realizado em terra e que estes tendem a aumentar a amplitude de movimento (ADM) das articulações, a força muscular, a capacidade cardiovascular e o equilíbrio postural refletindo, portanto, na melhora da capacidade funcional medida pelos instrumentos The Stanford Health AssessmentQuestionnaire(HAQ) ${ }^{(1,8,19)}$ The ArthritisImpactMeasurementScales 2 Questionnaire (AIMS 2) ${ }^{(3,11)}$ e FrenchayActivities Index (FAI). ${ }^{(1)}$ Essas melhoras funcionais devem aos princípios físicos da àgua que contribui na melhora da ADM através da força de flutuação facilitando o movimento articular, a viscosidade contribuindo na força muscular, a pressão hidrostática ajudando o condicionamento cardiovascular e melhorando com isso a funcionalidade do paciente com AR.Com relação à qualidade de vida encontrou-se que para avaliá-la, os autores têm feito uso, especialmente, dos questionários validadosShort Form-36 (SF-36) ${ }^{(2,3)}$ e EuroQol-5D (EQ5D). ${ }^{(8)}$ Além disso, foi possível evidenciar diminuição da dor mensurada pelas escalasVisual AnalogueScale (VAS), ${ }^{(1,8)}$ NumericalPain Rating Scale (NPRS) ${ }^{(14)}$ e questionários The Beliefs in PainControlQuestionnaire (BPCQ) $)^{(11)}$ e The McGillPainQuestionnaire (MPQ) ${ }^{(11)}$ na avaliação dos pacientes. Os pacientes de AR experimentam na piscina aquecida uma diminuição da sobrecarga articular que contribui na diminuição da dor e ajuda no relaxamento muscular.

\section{Capacidade Física}

Os pacientes portadores de $\mathrm{AR}$ apresentam um desempenho físico reduzido devido à dor, rigidez, inflamação articular, mobilidade articular diminuída e descondicionamento cardiovascular que comprometem a capacidade física e contribuem para a redução da qualidade de vida. Contudo, os exercícios físicos são indicados com intuito de melhorar os aspectos físicos, funcionais e psicológicos. Desta forma, os autores ao realizarem o tratamento aquático, verificaram a melhora da capacidade física dos pacientes com AR como ADM

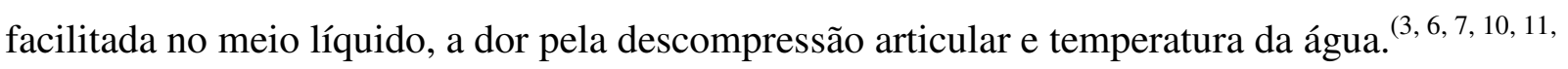
$17,18,21,22)$

Sandford-Smith, Mackay-Lyons \& Nunes-Clement ${ }^{(19)}$ realizaram um ensaio clínico com 24 indivíduos portadores de AR que foram divididos em dois grupos, um grupo realizou 
exercícios aeróbicos em piscina terapêutica e outro grupo submetido a um programa domiciliar de exercícios para ganho de ADM e fortalecimento. Ao observarem os resultados, constataram que houve melhora na habilidade da caminhada e valores do HAQ nos dois grupos, entretanto, o grupo que realizou exercícios para aumento da ADM e fortalecimento obteve melhores resultados estatisticamente. Os autores relataram que o grupo que se exercitou em piscina terapêutica apresentava faixa etária mais avançada comparados ao grupo submetido ao programa domiciliar. Isto pode ter interferido para obtenção de bons resultados. A força isométrica e isocinética do quadríceps femoral de oito participantes foi avaliada por Danneskiold-Samsoeet al., ${ }^{(6)}$ que realizaram um programa de exercícios por 10 semanas em piscina aquecida. Os autores observaram uma melhora da força isométrica e isocinética do quadríceps e, além disso, um aumento da capacidade aeróbica foi observado após o período de treinamento. $\mathrm{O}$ trabalho de força muscular com o paciente portador de AR na piscina terapêutica deve ser enfocado, já que a mesma é facilitada pela força de flutuação, a turbulência e a viscosidade, contribuindo de forma indireta para melhora da capacidade física. O paciente de AR tem uma facilidade em realizar o trabalho de força muscular na piscina por todo contexto já mencionados além das técnicas especificas como o BadRagaz e a Hidrocinesioterapiacontribuírem para o aumento da capacidade física.

Os pesquisadores Bilberg, Almén\&Mannerkorpi, ${ }^{(3)}$ realizaram um estudo controlado e randomizado com pacientes portadores de AR, no qual aplicaram exercícios aeróbicos em piscina aquecida num grupo de 20 indivíduos, o resultado de 24 sessões foi comparado com o grupo controle composto de 23 participantes que continuaram suas atividades prévias. Os pacientes submetidos a Fisioterapia Aquática melhoraram nos aspectos da função muscular tais como resistência isométrica dos ombros, força da garra, função muscular dos MMII e resistência dinâmica dos MMII avaliado pelo Chair Test que corresponde ao número de vezes que o indivíduo senta e levanta da cadeira durante um minuto. Além disso, os autores observaram uma melhora significativa na AIMS 2 e valor do HAQ. Tais resultados demonstram um aumento da capacidade física.A facilidade de movimento na piscina favorece a repetição do exercício, a pressão hidrostática a viscosidade e equipamentos aquáticos como a cama elástica contribuem na melhora da capacidade aeróbica do paciente e nas suas atividades de vida diária (AVD'S).

Em 1996, Hall et al. ${ }^{(11)}$ avaliaram a capacidade física e os aspectos emocionais de pacientes com AR ao aplicarem o instrumento AIMS 2 em quatro grupos. Dois grupos realizaram exercícios para ganho de ADM e força muscular em piscina terapêutica e em solo, 
e os outros fizeram relaxamento aquático e em solo por quatro semanas. Ao observarem os achados, constataram ganhos em todos os pacientes. Entretanto, após o período de tratamento aqueles que foram submetidos a Fisioterapia Aquática obtiveram resultados positivos clinicamente, demonstrando a contribuição da piscina terapêutica em seus aspectos de ganho de força muscular, ADM e relaxamento muscular, melhorando a capacidade física dos pacientes.

A capacidade física também foi verificada no estudo de Ritalaet al., ${ }^{(18)}$ que observaram ganhos de força muscular e mobilidade articular nos indivíduos que se exercitaram por 12 semanas num programa de exercícios aquáticos comparado a um grupo controle que realizou suas AVD'S. Desta forma, pode-se observar um aumento da capacidade funcional sem efeitos adversos.

$\mathrm{Na}$ análise de Dial \&Winson, ${ }^{(7)}$ mensuraram o ganho de ADM das articulações do cotovelo, punho, extensão das metacarpofalangeanas (MCF), quadril e tornozelo e realizaram testes cronometrados tais como levantar-sentar-andar, caminhada de 50 passos, colocar e retirar a camisa e duração da rigidez matinal. Além disso, o questionário de auto registro foi avaliado e logo após, os pacientes foram submetidos ao programa de exercícios aquáticos. Os participantes ficaram satisfeitos com o programa e também houve um aumento da força muscular e ADM das articulações do ombro, cotovelo, punho, quadril, joelho e melhor realização dos testes cronometrados. É importante ressaltar que neste estudo foi utilizado um número de pacientes pequeno, não podendo haver generalização. Contudo, os autores enfatizam a importância da Fisioterapia Aquática em pacientes com AR.

Em 1991, Stenströmet al. ${ }^{(21)}$ avaliaram os efeitos do treinamento intensivo dinâmico na água em 30 pacientes com AR e analisaram o resultado com o do grupo controle, com o mesmo número de pacientes durante quatro anos. Eles observaram que houve um aumento da atividade física e elevação da força muscular das mãos. Tais ganhos refletiram na melhora da capacidade funcional dos pacientes submetidos ao tratamento aquático.

No estudo de Melton-Rogers et al. ${ }^{(17)}$ compararam os exercícios em bicicleta ergométrica e a corrida na água com um aparelho de flutuação, sendo monitorizados o índice de percepção de esforço, freqüência cardíaca, freqüência respiratória, volume minuto, volume corrente, consumo de oxigênio e razão da troca respiratória. Os autores constataram que houve um aumento da razão de troca respiratória de pico, índice de percepção de esforço e pico da freqüência respiratória nos pacientes que realizaram corrida na água. O volume corrente apresentou maior valor nos pacientes que utilizaram bicicleta ergométrica. Desta forma, os 
resultados refletiram a melhora do condicionamento cardiovascular dos oito indivíduos que participaram do estudo.

Hall et al. ${ }^{(10)}$ observaram a caminhada em esteira aquática e esteira no solo de 15 mulheres que completaram três consecutivos turnos de caminhada de cinco minutos em 2,5 $\mathrm{km} / \mathrm{h}, 3,5 \mathrm{~km} / \mathrm{h}$ e $4,5 \mathrm{~km} / \mathrm{h}$. Eles verificaram o aumento da freqüência cardíaca e índice de percepção de esforço no solo e na água com aumento da velocidade. Além disso, velocidades abaixo de $3,5 \mathrm{~km} / \mathrm{h}$ o consumo de oxigênio foi maior na caminhada no solo que na água. A freqüência cardíaca diminuiu a velocidades abaixo de $2,5 \mathrm{~km} / \mathrm{h}$ na água similar em $3,5 \mathrm{~km} / \mathrm{h}$ e houve um aumento em $4,5 \mathrm{~km} / \mathrm{h}$. Os autores constataram que caminhada de $4,5 \mathrm{~km} / \mathrm{h}$ foi suficiente para aumentar a capacidade aeróbica.

Suomi\&Koceja, ${ }^{(22)}$ verificaram as medidas de desequilíbrio postural em 11 pacientes com osteoartrite (OA) e 13 indivíduos com AR, todos do sexo feminino sob um teste de apoio bipodal com visão e outro com apoio bipodal sem visão, administrado após um programa de exercícios aquáticos de seis semanas. Estes autores observaram que houve melhora do desequilíbrio postural lateral sob condições visuais após o tratamento, refletindo numa melhora da capacidade física.

Os efeitos do programa de Fisioterapia Aquática foram avaliados por Ahern et al. ${ }^{(1)} \mathrm{em}$ 1995, ao realizarem um estudo controlado e randomizado por seis semanas para pacientes com AR ou OA. O HAQ e FAI não indicaram melhoras consideráveis nos dois grupos. Todavia, ressalta-se que os pacientes apresentavam capacidade funcional limitada. Isto pode ter interferido os resultados.

\section{Qualidade de Vida}

Em geral, os pacientes com AR apresentam um decréscimo na qualidade de vida em consequiência da redução significativa da capacidade física e elevação dos níveis de dor. Dos estudos revisados, três avaliaram esta variável. ${ }^{(2,3,8)}$ No entanto, observou-se que por meio da aplicação dos questionários validados S-F36 ${ }^{(2,3)}$ e EQ-5D ${ }^{(8)}$ houve uma melhora da qualidade de vida.

O instrumento genérico da avaliação da saúde o SF-36 foi utilizado por Almeida, Netto\&Vinhas, ${ }^{(2)}$ em 2006, para mensurar a qualidade de vida de pacientes que receberam exercícios em solo comparados com aqueles que realizaram Fisioterapia Aquática. O grupo submetido aos exercícios em piscina terapêutica obteve um escore melhor nos itens aspectos 
emocionais e diminuição do tempo de rigidez matinal. Técnicas de relaxamento aquático como Watsu contribui no aspecto emocional pelo relaxamento globale melhora da mobilidade do paciente.Bilberg, Almén\&Mannerkorpi ${ }^{(3)}$ da mesma forma compararam um grupo que realizou exercícios de intensidade moderada em piscina terapêutica e um grupo controle que continuou suas atividades prévias. Os autores verificaram um aumento da qualidade de vida para o item vitalidade do SF-36 no grupo submetido ao tratamento aquático. Tais achados refletiram na melhora da qualidade de vida, entretanto, um número maior de pacientes seria necessário, para confirmar estatisticamente tais resultados. Desta forma estes achados não podem ser generalizados.

Eversdenet al. ${ }^{(8)}$ compararam os exercícios realizados em solo com a Fisioterapia Aquática e verificaram que não houve diferenças significantes entre os grupos de tratamento para o EQ-5D. Entretanto, os pacientes que realizaram os exercícios em piscina terapêutica, se sentiam muito melhores que aqueles submetidos ao tratamento em solo. Salienta-se que a utilização de diferentes fisioterapeutas pode ter levado a diferença dos resultados. Na avaliação final, três meses após completar o tratamento, muitos pacientes tinham modificado suas drogas anti-reumáticas modificadoras da doença (DMARDs) ou injeções de esteróides.

\section{Dor}

Clinicamente, a dor é um dos principais sintomas em patologias reumáticas, sendo relatada com frequiência em pacientes com AR. Desta forma, esta revisão, analisou estudos que verificaram a dor mensurada pela $\operatorname{VAS}^{(1,8)}$ e NPRS. ${ }^{(14)}$ Os questionários MPQ e BPCQ também foram utilizados. ${ }^{(11)}$ Em três artigos a redução da dor foi observada..$^{(1,11,14)}$

Numa pesquisa com 31 pessoas portadoras de AR e outras doenças reumáticas Lineker et al. ${ }^{(14)}$ determinaram a sensibilidade a mudanças nas medidas dos achados na aplicação da Fisioterapia Aquática por três meses. Eles observaram que a NPRS foi uma medida sensível a curto prazo. Os autores constataram uma diminuição do uso de medicamentos e redução das visitas aos profissionais de saúde. Os resultados refletiram na melhora da dor dos pacientes com doenças reumáticas.

Hall et al. ${ }^{(11)}$ observaram a redução da dor por meio da aplicação do questionário MPQ que avalia os aspectos quantitativos e qualitativos da dor e o questionário BPCQ nos quatro grupos do estudo. Entretanto, não foi possível verificar se a redução da dor foi mantida a longo 
prazo. Logo, a duração limitada do tratamento pode ter interferido para obtenção de melhores resultados.

Os pesquisadores Eversdenet al. ${ }^{(8)}$ avaliaram um grupo que realizou exercícios em solo e outro submetido ao tratamento aquático. Os autores verificaram que não houve diferenças significativas na VAS. Os pacientes tinham modificado suas DMARDs ou injeções de esteróides o que pode ter comprometido osresultados.

No estudo de Ahern et al. ${ }^{(1)}$ observaram um grupo composto de 22 pacientes que realizaram Fisioterapia Aquática e um grupo controle de oito participantes. Eles constataram por meio da VAS os pacientes com AR obtiveram melhores resultados que os participantes com OA na redução da dor. Todavia, faz-se interessante ressaltar que aspectos metodológicos podem ter influenciado nos resultados desse estudo já que o método VAS tem sido criticado na literatura.

\section{CONSIDERAÇÕES FINAIS}

Com base nas análises realizadas, conclui-se que a Fisioterapia Aquática pode beneficiar os pacientes portadores de AR com intuito de aumentar a ADM, força muscular, capacidade cardiovascular, funcionalidade, equilíbrio postural, reduzir a dor e assim proporcionar uma melhora na qualidade de vida dos indivíduos com AR. Recomenda-se que estes pacientes sejam estimulados a iniciar o tratamento em piscina aquecida precocemente para obtenção de melhores resultados.

Estudos com maior poder de gerar evidências são necessários para o aprofundamento de técnicas que poderão ser utilizadas no tratamento da referida patologia. E quanto a prática clínica, espera-se motivar os profissionais a explorar este recurso com embasamento e criatividade. Dessa forma, a Fisioterapia Aquática irá contribuir para que o paciente possa continuar a realização de suas habilidades funcionais.

\section{REFERÊNCIAS}

1. Ahern M, Nicholls E, Simionato E, Clark M, Bond M. Clinical and psychological effects of hydrotherapy in rheumatic diseases. ClinRehabil. 1995; 9: 204-12.

2. Almeida D, Netto KAR, Vinhas R. Estudo comparativo dos efeitos da fisioterapia aquática em relação à Fisioterapia em solo na qualidade de vida dos pacientes com artrite reumatóide. Rev FisioBrasil. 2006;79: 24-8. 
3. Bilberg A, Ahlmén M, Mannerkorpi K. Moderately intensive exercise in temperate pool for patients with rheumatoid arthritis: a randomized controlled study. Rheumatology. 2005;44(4): $502-8$.

4. Carvalho MAP, Xavier AJD. AJD. Artrite reumatóide. In: Moreira C, Carvalho MAP. Reumatologia diagnóstico e tratamento. 2a ed. Belo Horizonte: Medsi; 2001. p. 371-89.

5. Ciconelli RM. Artrite reumatóide. In: Sato E. Reumatologia: guias de medicina ambulatorial e hospital UNIFESP/ Escola Paulista de Medicina. São Paulo: Manole; 2004.p.85-97.

6. Danneskiold-Samsoe B, Lyngberg K, Risum T, Telling M. The effects of water exercise therapy given to patients with rheumatoid arthritis. Scand J Rehab Med. 1987; 19: 31-5.

7. Dial C, Windsor RA. A formative evaluation of a health education- water exercise program for class II and III adult rheumatoid arthritis patients. Patient EducCouns. 1985; 7: 33-42.

8. Eversden L, Maggs F, Nightingale P, Jobanputra P. A pragmatic randomized controlled trial of hydrotherapy and land exercises on overall well being and quality of life in rheumatoid arthritis. BMC Musculoskelet.Disord. 2007; 8(23):1-7.

9. Garret G. Aplicações Hidroterapêuticas na Reabilitação da Artrite. In: Becker, BE, Cole AJ. Terapia Aquática Moderna. São Paulo: Manole; 2000. p. 105-24.

10. Hall J,Grant J, Blake D, Taylor G, Garbutt G. Cardiorespiratory responses to aquatic treadmill walking in patients with rheumatoid arthritis. Physiother. Res. Int. 2004, 9(2): 59-73.

11. Hall J, Skevington SM, Maddison PJ, Chapman K. A randomized and controlled trial of hydrotherapy in rheumatoid arthritis.ArthCare Res. 1996; 9(3):206-15.

12. Laurindo IMM, Pinheiro GRC, Ximenes AC, Bertolo MB, Xavier RM, Giorgi RDN, et al. Consenso brasileiro para o dDiagnóstico e tratamento da artrite reumatóide.

Revbras.reumatol. 2002;42(6): 355-61.

13. Laurindo IMM, Tarigoe DY. Artrite reumatóide. In: Yoshinari NH, Bonfá ESDO. Reumatologia para o clínico. São Paulo: Roca; 2000. p.11-24.

14. LinekerSC, Badley EM, Hawker G, Wilkins A. Determining sensitivity to change in outcome measures used to evaluate hydrotherapy in rheumatic diseases. ArthCare Res.2000;13: 62-5.

15. Lloyd J. Artrite Reumatóide. In: David C, Lloyd J. Reumatologia para fisioterapeutas. São Paulo: EditoralPremier; 2001. p. 73-91.

16. McNeal R. Reabilitação aquática de pacientes com doença reumática. In: Ruoti RG, Morris DM, Cole AJ. Reabilitação aquática. São Paulo: Manole; 2000. p. 215-29. 
17. Melton-Rogers S, Hunter G, Walter J. Harrison P. Cardiorespiratory responses of patients with rheumatoid arthritis during bicycle riding and running in water.Phys.Ther. 1996; 76(10):1058-65.

18. Ritala P, Kettunen, McCubbin JA. Effects of water exercise program for individuals with rheumatoid arthitis. Sports Med Training and Rehab. 1996; 7:31-8.

19. Sandford-SmithS, Mackay-Lyons M, Nunes-Clement S. Therapeutic benefit of aquaerobics for individuals with rheumatoid arthritis. Physiother. Can.1998;Winter:40-6.

20. Skare TL. ArtriteReumatóide. In: Skare TL. Reumatologia princípios e prática. Rio de Janeiro: Guanabara Koogan; 1999. p. 91-104.

21. Stenström CH, Lindell B,Swanberg E, Swanberg P, Harms-Ringdahl K, Nordemar R. Intensive dynamic training in water for rheumatoid arthritis functional class II- a long term study of effects. Scand J Rheumatol. 1991; 20:358-65.

22. Suomi R, Koceja DM. Postural sway characteristics in women with lower extremity arthritis before and after an aquatic exercise intervention. Arch.Phys.Med. Rehabil. 2000; $81: 780-5$. 
Tabela 1: Características metodológicas e evidências observadas nos estudos para sistematizar o conhecimento acerca da capacidade física dos pacientes com AR.

\begin{tabular}{|c|c|c|c|c|}
\hline Autor/Ano & Delineamento do estudo & População do estudo & $\frac{\text { Variável analisada }}{\text { Capacidade física }}$ & Comentários \\
\hline $\begin{array}{c}\text { Danneskiold-Samsoeet al. } \\
1987\end{array}$ & Caso-Controle & 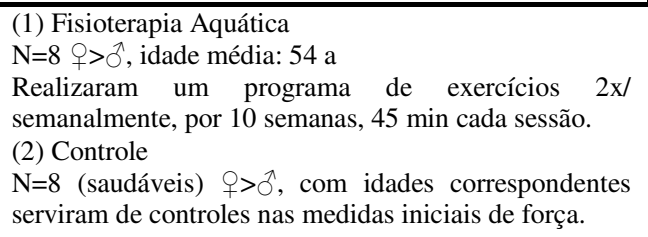 & $\begin{array}{l}\text { Foi avaliada a força isométrica e isocinética do } \\
\text { quadríceps. Houve melhora na média isométrica } \\
\text { máxima e força isocinética no pré-tratamento de } \\
88 \mathrm{~N} \text { e } 99 \mathrm{Nm} \text {. Após dois meses, a média isométrica } \\
\text { máxima e a força isométrica no quadríceps } \uparrow 38 \% \text { e } \\
16 \% \text { comparados ao pré-treinamento. }\end{array}$ & $\begin{array}{l}\text { Houve melhora da capacidade física em } \\
\text { todos os pacientes, exceto um que } \\
\text { desenvolveu arritmia cardíaca pelo teste de } \\
\text { capacidade aeróbica, o método Astrand. } \\
\uparrow \text { da capacidade aeróbica após o tratamento. }\end{array}$ \\
\hline $\begin{array}{l}\text { Melton-Rogers et al. } \\
1996\end{array}$ & Ensaio Clínico & $\begin{array}{l}\text { (1) Corrida de bicicleta estacionária + corrida na água } \\
\text { com aparelho de flutuação } \\
\mathrm{N}=8 \text { \&, idade média: } 35,88 \text { a } \\
\text { Peso: } 66.75 \mathrm{~kg} \\
\text { Altura média: } \approx 1.64 \mathrm{~m}\end{array}$ & $\begin{array}{l}\uparrow \text { da razão de troca respiratória de pico (24\%) água } \\
>\text { bicicleta } \\
\text { O índice de percepção de esforço (7\%) água > } \\
\text { bicicleta } \\
\uparrow \text { pico da FR (22\%) água > bicicleta. } \\
\uparrow \text { VC }(48 \%) \text { bicicleta >água }\end{array}$ & $\begin{array}{l}\text { Houve melhora da capacidade física, apesar } \\
\text { do } \mathrm{N} \text { pequeno. }\end{array}$ \\
\hline $\begin{array}{l}\text { Hall et al. } \\
2004\end{array}$ & Ensaio Clínico & $\begin{array}{l}\text { (1) Caminhada na esteira aquática + esteira em terra } \\
\mathrm{N}=15 \text {, idade média: } 47 \pm 8 \text { a } \\
\text { Peso: } 63.4 \mathrm{~kg} \\
\text { Altura média: } 1.62 \mathrm{~m} \\
\text { Completaram três turnos consecutivos com duração de } \\
5 \text { min de caminhada em } 2,5,3,5 \text { e } 4,5 \mathrm{~km} / \mathrm{h} \text { no solo e } \\
\text { em esteira aquática. }\end{array}$ & $\begin{array}{l}\uparrow F C \text { e índice de percepção de esforço no solo e na } \\
\text { água com } \uparrow \text { da velocidade } \\
\text { Abaixo de } 3,5 \mathrm{~km} / \mathrm{h} \text { o } \mathrm{VO} 2 \text { a caminhada no solo > } \\
\text { água. } \\
\downarrow \text { FC a } 2,5 \mathrm{~km} / \mathrm{h} \text { na água }>\text { caminhada no solo, } \\
\text { similar em } 3,5 \mathrm{~km} / \mathrm{h} \text { e } \uparrow \text { em } 4,5 \mathrm{~km} / \mathrm{h} \\
\uparrow \text { do índice de percepção de esforço na água } \\
\text { comparado ao solo. } \\
\text { VO2 } \approx 60 \% \text { do VO2 máx previsto durante a } \\
\text { velocidade mais rápida de caminhada na água. }\end{array}$ & $\begin{array}{l}\text { A caminhada de } 4,5 \mathrm{~km} / \mathrm{h} \text { foi suficiente para } \\
\text { estimar o } \uparrow \text { da capacidade aeróbica. }\end{array}$ \\
\hline
\end{tabular}




\begin{tabular}{|c|c|c|c|c|}
\hline Autor/Ano & Delineamento do estudo & População do estudo & $\begin{array}{c}\text { Variável analisada } \\
\text { Capacidade fisica } \\
\end{array}$ & Comentários \\
\hline $\begin{array}{l}\text { Dial, Windsor } \\
1985\end{array}$ & Ensaio Clínico & $\begin{array}{l}\mathrm{N}=12 \text { đ }>\text { P, idade média: } 59 \text { a } \\
\text {-Avaliação da ADM das art. do ombro, cotovelo, } \\
\text { punho, extensão MCF, quadril, tornozelo e testes } \\
\text { cronometrados, tais como, levantar-sentar-andar, } \\
\text { caminhada de } 50 \text { passos,colocar e retirar camisa, } \\
\text { duração da rigidez matinal. } \\
\text {-Questionário de auto registro, contendo } 28 \text { afirmativas } \\
\text { (habilidades de realizar AVD'S, percepções da } \\
\text { quantidade de dor nas articulações, quantidade de } \\
\text { rigidez e expectativas da eficácia do tratamento). } \\
\text { - Intervenção: } \\
\text { (1) Fisioterapia Aquática } \\
\text { 2x/ semanalmente, 1h cada sessão, durante oito } \\
\text { semanas. }\end{array}$ & $\begin{array}{l}\text { Os participantes ficaram satisfeitos com o programa } \\
\text { nos aspectos grupo e suporte social vindo de outros, } \\
\text { facilidade da atividade, liberdade temporária da dor, } \\
\uparrow \text { da mobilidade articular e força muscular. } \\
\uparrow \text { da ADM do ombro, cotovelo, punho, quadril, } \\
\text { joelho e testes cronometrados de levantar-sentar- } \\
\text { andar, caminhada de } 50 \text { passos e colocar e retirar a } \\
\text { camisa. }\end{array}$ & $\begin{array}{l}\text { Houve melhora considerável, apesar do } \mathrm{N} \\
\text { pequeno e falta do grupo controle. }\end{array}$ \\
\hline $\begin{array}{l}\text { Stenströmet al. } \\
1991\end{array}$ & Caso-Controle & $\begin{array}{l}\text { (1) Fisioterapia Aquática } \\
\mathrm{N}=30 \text { q }>\widehat{\jmath} \text {, idade média: } 52 \mathrm{a} \\
\text { Realizaram o treinamento } 1 \mathrm{x} / \text { semanalmente, } 40 \mathrm{~min} \text {, } \\
\text { durante quatro anos. } \\
\text { (2) Controle } \\
\mathrm{N}=30 \text { क }>\widehat{\delta} \text {, idade média: } 55 \mathrm{a}\end{array}$ & $\begin{array}{l}\text { Avaliados em relação às manifestações clínicas, } \\
\text { funcionais e consequiências psicossociais antes e } \\
\text { após o período de treinamento de quatro anos. } \\
\text { (1) apresenta } \uparrow \text { força muscular das mãos e } \uparrow \text { do nível } \\
\text { de atividade física. } \\
\uparrow \text { de admissão hospitalar em (2) durante o período } \\
\text { de treinamento. }\end{array}$ & $\begin{array}{l}\text { Bons resultados, } \uparrow \text { seus níveis de atividade } \\
\text { física. }\end{array}$ \\
\hline $\begin{array}{c}\text { Billberg, } \\
\text { Ahlmén\&Mannerkorpi } \\
2005\end{array}$ & $\begin{array}{l}\text { Ensaio Clínico Controlado } \\
\text { Randomizado }\end{array}$ & 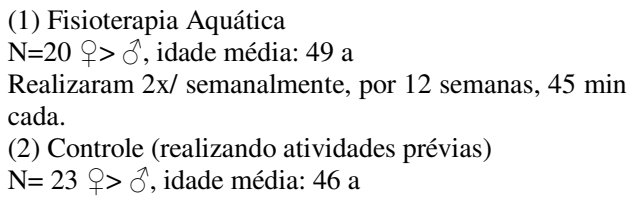 & $\begin{array}{l}\text { Houve melhoras significantes na resistência } \\
\text { isométrica dos m. abdutores do ombro, força da } \\
\text { garra, resistência dinâmica dos MMII (Chair Test) e } \\
\text { função muscular dos MMII. } \\
\uparrow \text { AIMS } 2 \\
\uparrow \text { HAQ }\end{array}$ & $\begin{array}{l}\text { Observou-se ganhos consideráveis apesar } \\
\text { do } \mathrm{N} \text { pequeno e as } \\
\text { conduzidas por dossos foram } \\
\text { alternados. }\end{array}$ \\
\hline
\end{tabular}




\begin{tabular}{|c|c|c|c|c|}
\hline Autor/Ano & Delineamento do estudo & População do estudo & $\frac{\text { Variável analisada }}{\text { Capacidade física }}$ & Comentários \\
\hline $\begin{array}{l}\text { Ritala et al } \\
1996\end{array}$ & Caso-Controle & 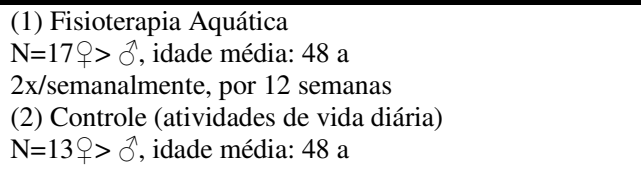 & $\begin{array}{l}(1)>(2) \text { na força muscular das mãos, mobilidade e } \\
\uparrow \text { da capacidade aeróbica. }\end{array}$ & $\begin{array}{l}\text { Houve melhoras significativas sem efeitos } \\
\text { deletérios. }\end{array}$ \\
\hline $\begin{array}{l}\text { Hall et al. } \\
1996\end{array}$ & $\begin{array}{l}\text { Ensaio Clínico Controlado } \\
\text { Randomizado }\end{array}$ & 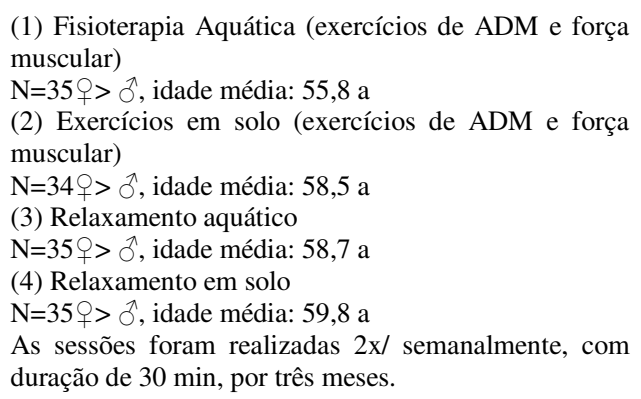 & $\begin{array}{l}\text { Os pacientes que realizaram atividades aquáticas } \\
\text { tiveram maior redução da sensibilidade articular } \\
\text { com uma média de } 27 \% \text { entre o pré e pós-teste. } \\
\uparrow \text { AIMS } 2 \text { em todos os pacientes } \\
\uparrow \text { ADM total de joelho em mulheres do grupo (1) }\end{array}$ & $\begin{array}{l}\text { Bons resultados, } \uparrow \text { capacidade física em } \\
\text { todos os pacientes. }\end{array}$ \\
\hline $\begin{array}{l}\text { Suomi\&Koceja } \\
\quad 2000\end{array}$ & Caso-Controle & $\begin{array}{l}\text { (1) Fisioterapia Aquática } \\
\mathrm{N}=7 \mathrm{AR} \text { } \\
\mathrm{N}=7 \mathrm{OA} \\
\text { (2) Controle } \\
\mathrm{N}=4 \mathrm{AR} \text { Idade média: } 60,7 \mathrm{a} \\
\mathrm{N}=6 \mathrm{OA} 9 \\
\text { Realizaram o teste de apoio bipodal nas condições de } \\
\text { olhos abertos e olhos fechados. Em cada dia de teste, as } \\
\text { participantes realizaram um bloco de seis tarefas de } \\
15 \text { " separadas por 30" de descanso entre cada } \\
\text { experimento. } \\
\text { Os exercícios aquáticos foram realizados } 3 \mathrm{x} / \\
\text { semanalmente, com duração de } 45 \text { min, por seis } \\
\text { semanas. }\end{array}$ & $\begin{array}{l}\text { (1) }>\text { (2) } \downarrow \text { significativa no desequilíbrio postural } \\
\text { lateral. Além disso, houve } \downarrow \text { das áreas de } \\
\text { desequilíbrio de } 18 \% \text { a } 30 \% \text { em apoio bipodalsob } \\
\text { condições visuais após a intervenção. }\end{array}$ & $\begin{array}{l}\text { Verificou-se que as medidas de } \\
\text { desequilíbrio postural em mulheres com AR } \\
\text { e OA sob teste estável de apoio bipodal } \\
\text { pode ser confiável, seguro e de fácil } \\
\text { realização. }\end{array}$ \\
\hline
\end{tabular}




\begin{tabular}{|c|c|c|c|c|}
\hline Autor/Ano & Delineamento do estudo & População do estudo & $\frac{\text { Variável analisada }}{\text { Capacidade física }}$ & Comentários \\
\hline $\begin{array}{l}\text { Eversdenet al. } \\
\quad 2007\end{array}$ & $\begin{array}{l}\text { Ensaio Clínico Controlado } \\
\text { Randomizado }\end{array}$ & 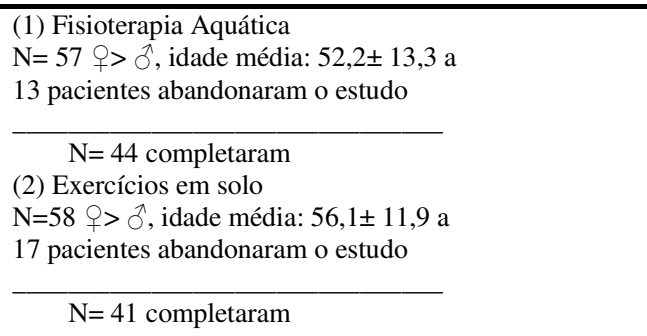 & $\begin{array}{l}\text { Os pacientes do grupo (1) sentem muito melhores } \\
\text { que (2), porém não houve diferenças significativas } \\
\text { entre os grupos (1) e (2) para o HAQ. }\end{array}$ & $\begin{array}{l}\text { A utilização de diferentes fisioterapeutas } \\
\text { pode ter levado a diferença dos resultados. } \\
\mathrm{Na} \text { avaliação final, três meses após } \\
\text { completar o tratamento muitos pacientes } \\
\text { tinham modificado ou } \uparrow \text { suas DMARDs ou } \\
\text { injeções de esteróides. }\end{array}$ \\
\hline $\begin{array}{l}\text { Ahern et al. } \\
1995\end{array}$ & $\begin{array}{l}\text { Ensaio Clínico Controlado } \\
\text { Randomizado }\end{array}$ & $\begin{array}{l}\text { (1) Fisioterapia Aquática } \\
\text { - Estudo de quatro dias: } \\
\mathrm{N}=32 \mathrm{AR} \\
\mathrm{N}=40 \mathrm{OA} \\
\hat{0}>\varnothing \text {; idade média: } 66,9 \text { a } \\
\text { - Estudo de seis semanas: } \\
\mathrm{N}=10 \mathrm{AR} \\
\mathrm{N}=20 \mathrm{OA} \\
\hat{O}>\varnothing ; \text { idade média: } 67,7 \text { a } \\
\text { Após os quatro dias, } 22 \text { pacientes foram tratados com } \\
\text { Fisioterapia Aquática contínua e oito pacientes } \\
\text { terminaram o tratamento e atuaram como controle. }\end{array}$ & $\begin{array}{l}\text { O HAQ e FAI foram avaliados no estudo de seis } \\
\text { semanas. Não houve efeitos significativos do FAI e } \\
\text { HAQ. }\end{array}$ & $\begin{array}{l}\text { O tempo de intervenção e o } \mathrm{N} \text { pequeno } \\
\text { podem ter sido fatores importantes que } \\
\text { interferiram nos resultados }\end{array}$ \\
\hline $\begin{array}{c}\text { Sandford - Smith, Mackay- } \\
\text { Lyons \&Nunes-Clement } \\
1998\end{array}$ & Ensaio Clínico & 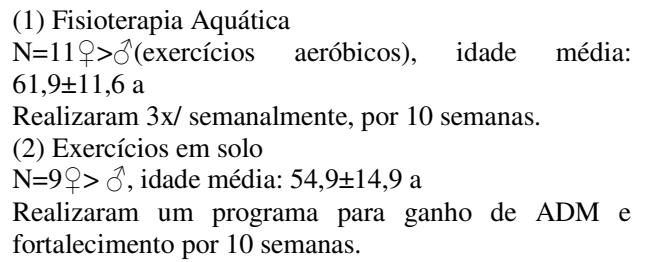 & (2) $>$ (1) nos valores do HAQ & $\begin{array}{l}\mathrm{N} \text { pequeno podem ter sido fator importante } \\
\text { que interferiu nos resultados. }\end{array}$ \\
\hline
\end{tabular}




\begin{tabular}{|c|c|c|c|c|}
\hline Autor/Ano & Delineamento do estudo & População do estudo & $\begin{array}{l}\text { Variável analisada } \\
\text { Qualidade de vida } \\
\end{array}$ & Comentários \\
\hline $\begin{array}{l}\text { Almeida, Netto \&Vinhas } \\
2006\end{array}$ & Caso-controle & $\begin{array}{l}\text { (1) Fisioterapia Aquática } \\
\mathrm{N}=15 \text {, idade média: } 43,73 \text { a } \\
\text { Sendo }+>\hat{0} \\
\text { (2) Exercícios em solo } \\
\mathrm{N}=15 \text { q, idade média: } 51,27 \text { a }\end{array}$ & $\begin{array}{l}\text { Pelo SF-36 (1) > (2) após o tratamento nos itens } \\
\text { aspectos emocionais e diminuição do tempo de } \\
\text { rigidez matinal. } \\
\text { (2) }>\text { (1) para o item capacidade funcional. }\end{array}$ & $\begin{array}{l}\text { Observou-se ganhos significativos } \\
\text { apesar do N pequeno e a falta do } \mathrm{n}^{\circ} \text { de } \\
\text { sessões, duração, frequêencia nos dois } \\
\text { grupos. }\end{array}$ \\
\hline $\begin{array}{l}\text { Eversdenet al. } \\
\quad 2007\end{array}$ & $\begin{array}{l}\text { Ensaio Clínico Controlado } \\
\text { Randomizado }\end{array}$ & $\begin{array}{l}\text { (1) Fisioterapia Aquática } \\
\mathrm{N}=57 \text { }>>\widehat{\partial} \text {, idade média: } 52,2 \pm 13,3 \text { a } \\
13 \text { pacientes abandonaram o estudo } \\
\mathrm{N}=44 \text { completaram } \\
\text { (2) Exercícios em solo } \\
\mathrm{N}=58 \text { }>\widehat{O} \text {, idade média: } 56,1 \pm 11,9 \text { a } \\
17 \text { pacientes abandonaram o estudo }\end{array}$ & $\begin{array}{l}\text { Os pacientes do grupo (1) sentem muito melhores } \\
\text { que (2), porém não houve diferenças significativas } \\
\text { entre os grupos (1) e (2) para o EQ-5D. }\end{array}$ & $\begin{array}{l}\text { A utilização de diferentes } \\
\text { fisioterapeutas pode ter levado a } \\
\text { diferença dos resultados. } \\
\text { Na avaliação final, três meses após } \\
\text { completar o tratamento muitos } \\
\text { pacientes tinham modificado ou } \uparrow \text { suas } \\
\text { DMARDs ou injeções de esteróides. }\end{array}$ \\
\hline & & $\mathrm{N}=41$ completaram & & \\
\hline $\begin{array}{l}\text { Billberg, } \\
\text { Ahlmén\&Mannerkorpi } \\
2005\end{array}$ & $\begin{array}{l}\text { Ensaio Clínico Controlado } \\
\text { Randomizado }\end{array}$ & 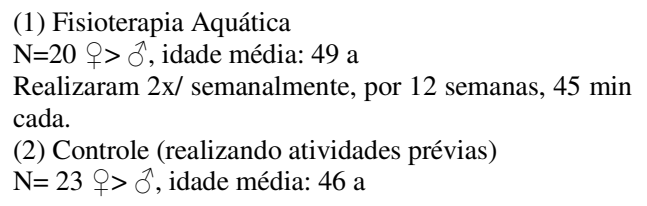 & $\begin{array}{l}\text { Pelo SF-36 houve melhoras significantes em (1)>(2) } \\
\text { no item vitalidade. }\end{array}$ & $\begin{array}{l}\text { Ganhos consideráveis apesar do } \mathrm{N} \\
\text { pequeno e as sessões foram } \\
\text { conduzidas por dois fisioterapeutas } \\
\text { alternados. }\end{array}$ \\
\hline $\begin{array}{l}\text { Lineker et al. } \\
\quad 2000\end{array}$ & Ensaio Clínico & $\begin{array}{l}\text { (1) Fisioterapia Aquática } \\
\mathrm{N}=31 \text { \% , idade média }=57 \text { a } \\
\text { Sendo } \mathrm{N}=32,3 \% \quad \mathrm{AR} ; \quad \mathrm{N}=41,9 \% \quad \mathrm{OA} ; \mathrm{N}=25,8 \% \\
\text { Fibromialgia; } \mathrm{N}=19,2 \% \text { outros. } \\
\text { Foram avaliados antes, em } 10 \text { semanas, e três meses } \\
\text { após o programa. }\end{array}$ & $\begin{array}{l}67 \% \text { dos pacientes registraram melhoras no } \\
\text { componente dor no SF-36. }\end{array}$ & $\begin{array}{l}\text { Observou-se a falta de um grupo } \\
\text { controle, duração de sessões e } \\
\text { freqüência. }\end{array}$ \\
\hline
\end{tabular}




\begin{tabular}{|c|c|c|c|c|}
\hline Autor/Ano & Delineamento do estudo & População do estudo & $\begin{array}{c}\text { Variável analisada } \\
\text { Dor }\end{array}$ & Comentários \\
\hline $\begin{array}{l}\text { Ahern et al. } \\
1995\end{array}$ & $\begin{array}{l}\text { Ensaio Clínico Controlado } \\
\text { Randomizado }\end{array}$ & $\begin{array}{l}\text { (1) Fisioterapia Aquática } \\
\text { - Estudo de quatro dias: } \\
\mathrm{N}=32 \mathrm{AR} \\
\mathrm{N}=40 \mathrm{OA} \\
\hat{0}>\text { O } ; \text {; idade média: } 66,9 \text { a } \\
\text { Após os quatro dias, } 22 \text { pacientes foram tratados com Fisioterapia Aquática contínua e } \\
\text { oito pacientes terminaram o tratamento e atuaram como controle. } \\
\text { - Estudo de seis semanas: } \\
\mathrm{N}=10 \mathrm{AR} \\
\mathrm{N}=20 \mathrm{OA} \\
\delta>\varnothing ; \text { idade média: } 67,7 \text { a }\end{array}$ & $\begin{array}{l}\text { No estudo de quatro dias houve } \\
\text { melhora na auto - eficácia da } \\
\text { dor, porém } A R>\text { OA. } \\
\downarrow \text { VAS AR }>\text { OA. }\end{array}$ & $\begin{array}{l}\text { Observou-se melhoras consideráveis } \\
\text { da dor nos pacientes com AR e OA. }\end{array}$ \\
\hline $\begin{array}{l}\text { Eversdenet al. } \\
\quad 2007\end{array}$ & $\begin{array}{l}\text { Ensaio Clínico Controlado } \\
\text { Randomizado }\end{array}$ & 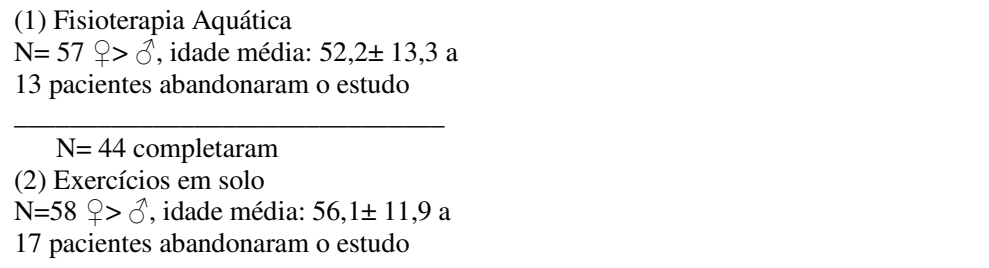 & $\begin{array}{l}\text { Não houve melhoras } \\
\text { significativas na dor VAS. }\end{array}$ & $\begin{array}{l}\text { A utilização de diferentes } \\
\text { fisioterapeutas pode ter levado a } \\
\text { diferença dos resultados. } \\
\text { Na avaliação final, três meses após } \\
\text { completar o tratamento muitos } \\
\text { pacientes tinham modificado ou } \uparrow \text { suas } \\
\text { DMARDs ou injeções de esteróides. }\end{array}$ \\
\hline & & $\mathrm{N}=41$ completaram & & \\
\hline $\begin{array}{l}\text { Hall et al. } \\
1996\end{array}$ & $\begin{array}{l}\text { Ensaio Clínico Controlado } \\
\text { Randomizado }\end{array}$ & 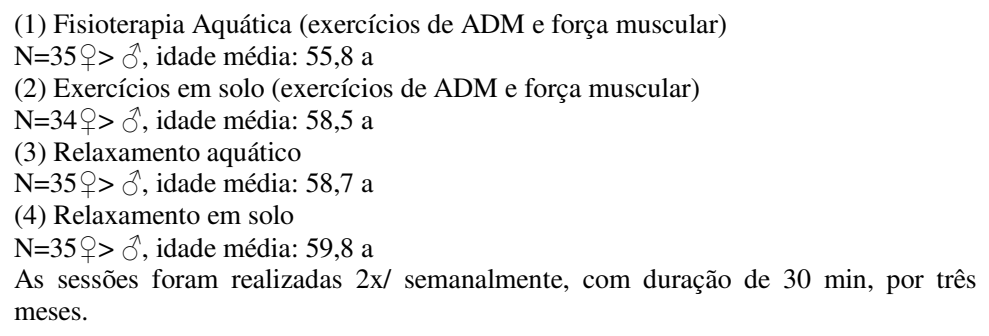 & $\begin{array}{l}\downarrow \text { MPQ e BPCQ nos quatro } \\
\text { grupos do estudo. }\end{array}$ & $\begin{array}{l}\text { Bons resultados, entretanto, os } \\
\text { resultados não foram mantidos a longo } \\
\text { prazo. }\end{array}$ \\
\hline $\begin{array}{l}\text { Lineker et al. } \\
\quad 2000\end{array}$ & Ensaio Clínico & $\begin{array}{l}\text { (1) Fisioterapia Aquática } \\
\text { N=31 } 9 \text {, idade média: } 57 \text { a } \\
\text { Sendo N=32,3\% AR; N= } 41,9 \text { OA; N=25,8\% Fibromialgia; N=19,2\% outros. } \\
\text { Foram avaliados antes, em } 10 \text { semanas, e três meses após o programa. }\end{array}$ & $\begin{array}{l}\text { A NPRS foi considerada medida } \\
\text { sensível a curto prazo. }\end{array}$ & $\begin{array}{l}\text { Observou-se a falta de um grupo } \\
\text { controle, duração de sessões e } \\
\text { frequiência. }\end{array}$ \\
\hline
\end{tabular}

